\title{
Scalable and selective $\beta$-hydroxy- $\alpha$-amino acid synthesis catalyzed by promiscuous L-threonine transaldolase ObiH
}

\author{
Tyler J. Doyon, ${ }^{\ddagger[a]}$ Prasanth Kumar, ${ }^{\ddagger[a]}$ Sierra Thein, ${ }^{[b]}$ Maeve Kim, ${ }^{[b]}$ Abigail Stitgen, ${ }^{[b]}$ Abbigail M. \\ Grieger, ${ }^{[b]}$ Cormac Madigan, ${ }^{[b]}$ Patrick H, Willoughby, ${ }^{*[b]}$ and Andrew R. Buller ${ }^{*[a]}$
}

[a] Dr. T.J. Doyon, Prasanth Kumar, Prof. A.R. Buller Department of Chemistry

University of Wisconsin-Madison

Madison, WI 53706, United States

E-mail: arbuller@wisc.edu

[b] S. Thein, M. Kim, A. Stitgen, A. Grieger, C. Madigan, Prof. P.H. Willoughby

Department of Chemistry

Ripon College

Ripon, WI 54971, United States

* $\quad$ Corresponding author

$\ddagger \quad$ Designates equal contribution

Supporting information for this article is given via a link at the end of the document

\begin{abstract}
Enzymes from secondary metabolic pathways possess broad potential for the selective synthesis of complex bioactive molecules. However, the practical application of these enzymes for organic synthesis is dependent on the development of efficient, economical, operationally-simple and wellcharacterized systems for preparative scale reactions. We sought to bridge this knowledge gap for the selective biocatalytic synthesis of $\beta$-hydroxy- $\alpha$-amino acids, which are important synthetic building blocks. To achieve this goal, we demonstrated the ability of $\mathrm{ObiH}$, an L-threonine transaldolase, to achieve selective gram-scale synthesis of a diverse array of nonstandard amino acids (nsAAs) using a scalable whole cell platform. We show how the initial selectivity of the catalyst is high and how the diastereomeric ratio of products decreases at high conversion due to product re-entry into the catalytic cycle. ObiH-catalyzed reactions with a variety of aromatic, aliphatic and heterocyclic aldehydes selectively generated a panel of $\beta$ hydroxy- $\alpha$-amino acids possessing broad functional-group diversity. Furthermore, we demonstrated that ObiH-generated $\beta$ hydroxy- $\alpha$-amino acids could be modified through additional transformations to access important motifs, such as $\beta$-chloro- $\alpha$ amino acids and substituted $\alpha$-keto acids.
\end{abstract}

\section{Introduction}

Nature has evolved powerful and effective approaches to selectively generate complex natural products from simple metabolites. ${ }^{[1]}$ Biosynthetic enzymes often catalyze transformations that are challenging to achieve using small molecule methods and can enable efficient access to valuable molecules. ${ }^{[2]}$ In comparison to traditional synthetic transformations, the three-dimensional architecture of enzyme active sites enables exquisite control over the positioning of reactants, often leading to improved chemo-, site- and stereoselectivity profiles. As a result, a wide variety of new biocatalytic methods have been developed in recent years which seek to resolve selectivity challenges in complex molecule synthesis. ${ }^{[3]}$ Functional group interconversions are the principle class of organic transformations for which high quality biocatalytic routes have been successfully developed. ${ }^{[3]}$ In contrast, the production of novel biocatalytic methods for stereocontrolled $\mathrm{C}-\mathrm{C}$ bond formation remains a promising, but underexplored area, with many exciting new advances underway. ${ }^{[4,5]}$ The ability to selectively and efficiently construct a carbon framework enables concise access to target molecules, improving the sustainability and reducing the environmental impact of synthetic routes. Biocatalytic approaches for $\mathrm{C}-\mathrm{C}$ bond formation possess unique potential for streamlining the construction of complex molecular building blocks, natural products, and pharmaceuticals. ${ }^{[4,6]}$ We envisioned that one such important class of molecules, $\beta$-hydroxy- $\alpha$-amino acids, could be selectively generated in an efficient and highly scalable fashion by designing and employing a biocatalytic reaction platform.

$\beta$-Hydroxy- $\alpha$-amino acids are a class of molecules commonly found in polypeptide natural products and pharmaceuticals (Figure 1A). ${ }^{[1]}$ In particular, these molecules are prominent intermediates in the biosynthesis of cyclic peptide and small molecule antibiotics, such as the compounds hypeptin (1), katanosin B (2), and chloramphenicol (4), as well as other bioactive molecules. ${ }^{[7-9]}$ Motivated by these important structures, researchers have developed a variety of organocatalytic methods to install the $\beta$-hydroxyl group, often via the aldol addition of a glycyl Ca-nucleophile into various aldehydes. ${ }^{[10-15]}$ These methods are effective at generating $\beta$-hydroxy amino acids, but often require orthogonal, multi-step protecting group strategies to produce the desired product which can increase costs and reduce overall yields. Consequently, researchers have sought to develop biocatalytic approaches to $\beta$-hydroxy amino acid synthesis, which offer improved selectivity, use aqueous solvents, and utilize sustainably produced catalysts that can be deployed at low concentrations.

In Nature, the $\beta$-hydroxy functional group is often installed on a pre-assembled amino acid through site-selective oxidation by cytochromes P450 or a-ketoglutarate dependent non-heme iron enzymes. ${ }^{[16-19]}$ However, the ability to assemble the side chain while simultaneously setting the stereochemistry of the $\beta$ hydroxy group offers a more versatile, complementary approach for the preparative scale synthesis of structurally diverse nsAAs. Researchers have therefore been drawn to the pyridoxal phosphate (PLP)-dependent threonine aldolases (TAs) and 


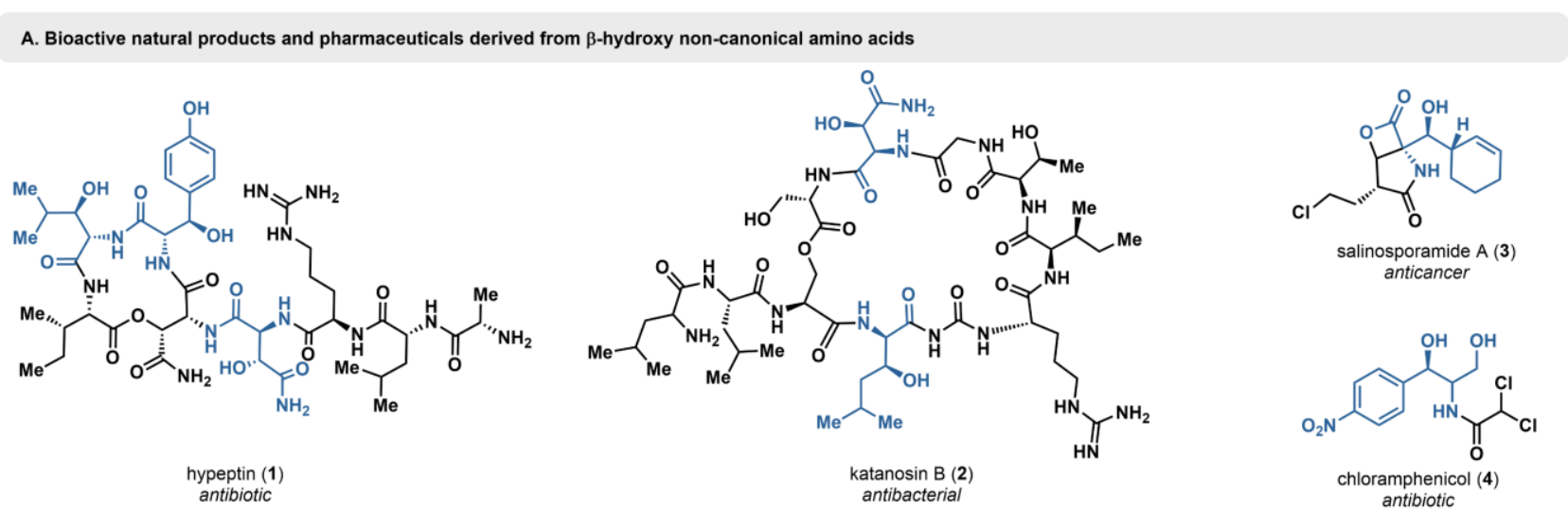

B. ObiH-catalyzed native $\mathrm{C}-\mathrm{C}$ bond formation in obafluorin biosynthesis

C. L-threonine transaldolase-catalyzed synthesis of benzylic $\beta$-hydroxy amino acids

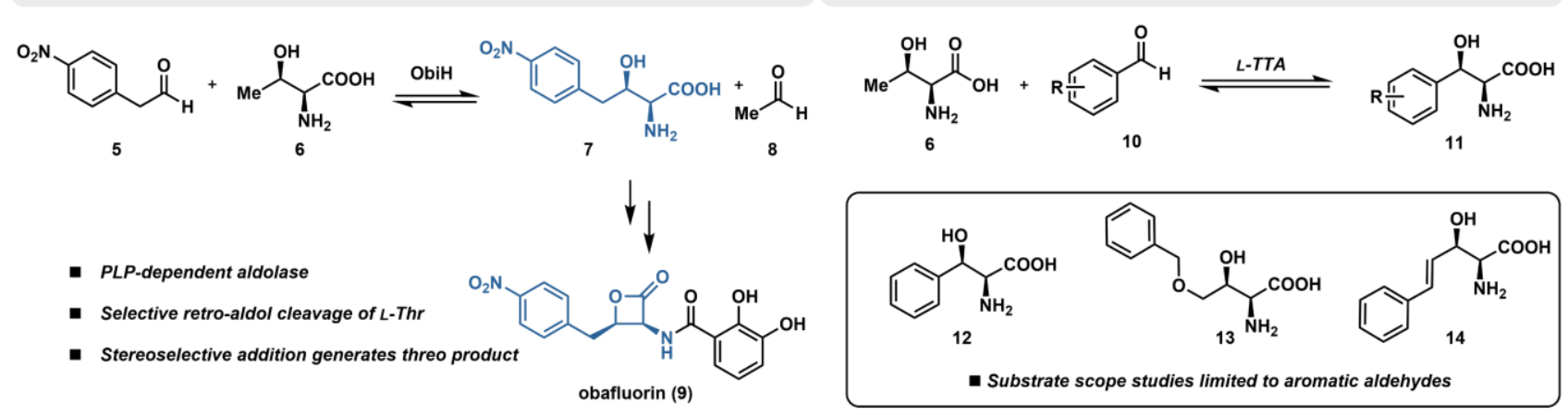

D. This work: scalable and efficient production of diverse $\beta$-hydroxy non-canonical amino acids using whole cell biocatalysis

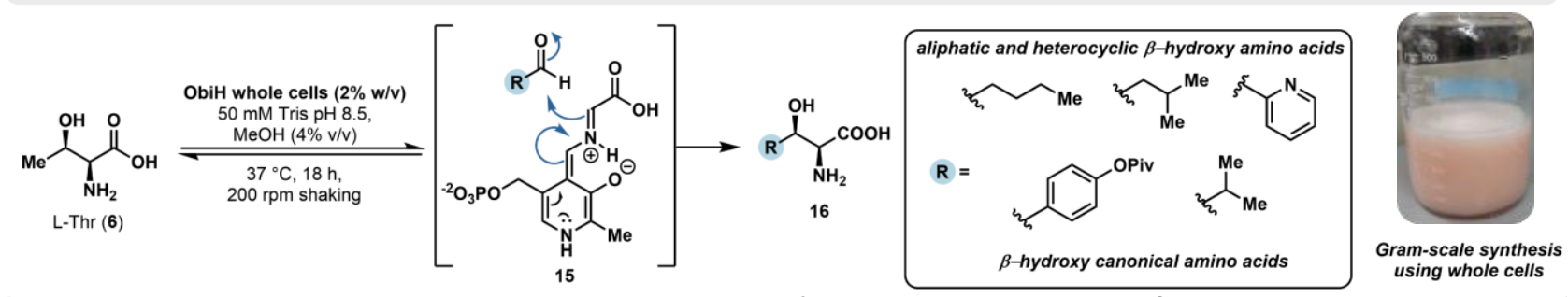

Figure 1. A. Bioactive natural products and pharmaceuticals containing $\beta$-hydroxy- $\alpha$-amino moieties. $\mathrm{B}$. ObiH native reaction in the context of obafluorin biosynthesis. C. Previous substrate scope studies using L-threonine transaldolases (LTTAs). D. This work: highly scalable and efficient generation of diverse $\beta$-hydroxy- $\alpha$-amino acids.

serine hydroxymethyl transferases (SHMTs) for synthesis of $\beta$ These enzymes natively catalyze the retro-aldol cleavage of Lthreonine (Thr) and L-serine (Ser), respectively, generating glycine for use in primary metabolism. ${ }^{[21]}$ By running these transformations in reverse with very high concentrations of glycine, TAs and SHMTs have been leveraged for the synthesis of $\beta$-hydroxy- $\alpha$-amino acids. However, most native TAs catalyze aldol reactions with low stereoselectivity at $C \beta$, leading to poor diastereoselectivity profiles for non-native transformations. ${ }^{\text {[22-25] }}$ These challenges can be overcome in exceptional cases, either through intensive directed evolution or through the use of diastereoselective crystallization, but these routes are limited to one or a handful of substrates before selectivity is compromised. ${ }^{[23,26]}$ Consequently, a scalable and generalizable biocatalytic route for the selective synthesis of $\beta$-hydroxy amino acids has yet to be realized.

An alternative and appealing enzymatic strategy for the synthesis of $\beta$-hydroxy amino acids draws directly from Nature's own approach. Rather than contend with the endergonic challenge of reversing the TA reaction, there are a set of hydroxy amino acids. ${ }^{[20]}$ mechanistically-related L-threonine transaldolase (LTTA) enzymes that catalyze removal of the Thr sidechain as acetaldehyde and its replacement with a new aldehyde (5) to generate a new $\beta$-hydroxy amino acid (Figure 1B). This reaction does not release Gly as an intermediate and is netthermoneutral. High yields are enabled by coupling to downstream metabolism. ${ }^{[27,28]}$ Notable among LTTA enzymes is $\mathrm{ObiH}$, which catalyzes the diastereoselective synthesis of $\beta$ hydroxy amino acid 7 in the biosynthesis of the aminoacyl tRNA synthetase inhibitor obafluorin (Figure 1B, 9). ${ }^{[27-29]}$ Previous efforts to utilize LTTAs for synthetic purposes focused on optimization of analytical scale reactions with electron-deficient aromatic aldehydes (Figure 1C). ${ }^{[29,30]}$ Protein engineering has also been deployed to increase reactivity with $p$ methylsulfonylbenzaldehyde en route to the antibiotic thiamphenicol.. ${ }^{[31]}$ Mirroring Nature's approach, preparative scale LTTA transformations with this substrate were driven to high yield by coupling to a downstream enzymatic reduction of acetaldehyde. ${ }^{[30]}$ These promising results prompted deeper 
study to understand the difference in reactivity between TA and LTTA enzymes, which we recently addressed through a detailed

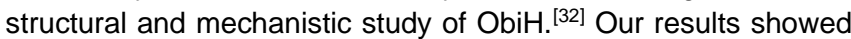
that the nucleophilic intermediate, a PLP bound glycyl quinonoid, $E\left(Q^{G l y}\right) 15$, is formed by retro-aldol cleavage of Thr. Further spectroscopic analysis showed this intermediate is kinetically shielded from protonation. Monitoring persistence of the $E\left(Q^{\mathrm{Gly}}\right)$ in the presence of small aliphatic aldehydes indicated that $\mathrm{ObiH}$ has reactivity toward these electrophiles, which we confirmed via synthesis of $\beta$-hydroxyl-L-leucine using purified $\mathrm{ObiH}^{\left[{ }^{[32]}\right.}$ Translating these exciting advances in enzymology to a practical biocatalytic route remains, however, a separate challenge.

For facile adoption by the synthetic community, an LTTA route to $\beta$-hydroxy amino acids must be deployed in the most operationally-simple fashion and the limitations of the aldehyde scope established. It has been previously observed that the diastereomeric ratio $(\mathrm{dr})$ of the products of LTTA reactions decrease through an unknown mechanism, confounding the reproducibility of stereochemical outcomes. ${ }^{[33]}$ Furthermore, biocatalytic methods often yield unprotected, hydrophilic products and demonstration of standardized methods for their isolation and downstream manipulation further increase the attractiveness of an enzymatic route. Here, we tackle these challenges and demonstrate how $\mathrm{ObiH}$ be efficiently prepared for, and utilized as, an effective catalyst for the synthesis of structurally diverse $\beta$-hydroxy amino acids from inexpensive and readily available starting materials (Figure $1 \mathrm{D}$ ).

\section{Results and Discussion}

We sought to develop a scalable and operationally-simple method for the selective synthesis of a variety of $\beta$-hydroxy- $\alpha$ amino acids. To meet this objective, we reasoned that using a well-studied LTTA as the biocatalyst would provide an ideal starting point for method development. ObiH is a structurally and mechanistically characterized enzyme that has been shown to possess high transaldolase activity with several electron-poor aldehydes. ${ }^{[27,28,32]}$ We first attempted reactions using wet, whole $E$. coli cells that contained heterologously-expressed $\mathrm{N}$-HisObiH. Biocatalytic transformations using whole cells have been demonstrated as an economical and efficient route to access valuable molecules without the need for time and resource-

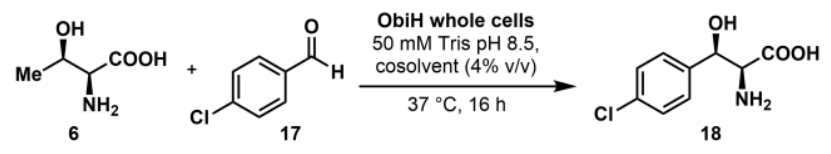

\begin{tabular}{cccc}
\hline entry & whole cell concentration $(\%)$ & cosolvent & percent conversion \\
\hline 1 & $0.5 \%(5 \mathrm{mg} / \mathrm{mL})$ & MeOH & $88 \%$ \\
2 & $0.5 \%(5 \mathrm{mg} / \mathrm{mL})$ & no cosolvent & $86 \%$ \\
3 & $0.5 \%(5 \mathrm{mg} / \mathrm{mL})$ & toluene & $82 \%$ \\
4 & $0.5 \%(5 \mathrm{mg} / \mathrm{mL})$ & $\mathrm{MeCN}$ & $69 \%$ \\
5 & $1.0 \%(10 \mathrm{mg} / \mathrm{mL})$ & $\mathrm{MeOH}$ & $90 \%$ \\
6 & $2.0 \%(20 \mathrm{mg} / \mathrm{mL})$ & $\mathrm{MeOH}$ & $91 \%$ \\
7 & $4.0 \%(40 \mathrm{mg} / \mathrm{mL})$ & $\mathrm{MeOH}$ & $88 \%$ \\
\hline
\end{tabular}

Table 1. Analytical scale optimization of ObiH-catalyzed aldol reactions with 4-chlorobenzaldehyde (17). Conversion values are reported as the average of duplicate experiments. intensive enzyme purification procedures. ${ }^{[34-36]}$ Initial optimization reactions with 4-chlorobenzaldehyde (17) revealed that $\mathrm{ObiH}$ was catalytically active in the whole cell format (Table 1 , entry 1), providing product 18 with $88 \%$ conversion, as measured by UPLC-MS. We screened alternative cosolvent compositions at a whole cell concentration of $5 \mathrm{mg} / \mathrm{mL}(0.5$ wt \%). In general, there was only a modest impact on reactivity at this cell loading. $\mathrm{MeOH}$ and toluene $(4 \% \mathrm{v} / \mathrm{v})$ each had no significant effect on activity when compared to reactions lacking cosolvent (Table 1, entries 1-3), whereas MeCN was detrimental to enzyme activity. Further optimization of wet whole cell loading was performed using $\mathrm{MeOH}$ as a cosolvent (Table 1, entries 5$7)$. Increasing whole cell loadings as high as $4 \%(20 \mathrm{mg} / \mathrm{mL})$ maintained high conversions. Anticipating that alternative electrophiles may not react as efficiently with $\mathrm{ObiH}$, we elected to use $1-2 \%$ wet whole cells and $4 \%(\mathrm{v} / \mathrm{v}) \mathrm{MeOH}$ (Table 1, entry 6) for future applications as it can deliver high substrate conversions (>90\%) without excessive catalyst and with an easy-to-remove co-solvent. Cell loadings of this magnitude are sufficiently low for process scale reactions and are often only achieved through extensive protein engineering. We attribute this excellent reactivity of $\mathrm{ObiH}$ to its high soluble expression in E. coli, boasting $>150 \mathrm{mg}$ protein per $\mathrm{L}$ culture.

With these conditions in hand, we were motivated to explore the limits of ObiH-catalyzed reactions by probing the native substrate promiscuity of the catalyst, with the goal to selectively generate and isolate a diverse array of syntheticallyand pharmaceutically-valuable $\beta$-hydroxy- $\alpha$-amino acids. Previous efforts with an ObiH homolog focused on the analytical scale synthesis of phenylserine derivatives, with a single example of product isolation. ${ }^{[30]}$ To gain a more comprehensive view of ObiH reactivity, we chose to perform analytical and preparative scale reactions and compare the relative yields and observed selectivities. Under our standardized reaction conditions, unprotected $\beta$-hydroxy amino acids with halogen functionalities were swiftly generated in high yields on analytical scale, but with low to moderate diastereoselectivity as calculated by UPLC-PDA-MS analysis after functionalization with Marfey's reagent (Figure 2). In comparison, preparative scale reactions with the same substrates delivered products with enhanced diastereoselectivity, albeit with reduced yields. These data suggest that $\mathrm{ObiH}$ is subject to a selectivity phenomenon observed with other LTTAs. ${ }^{[33,37]}$ Namely, an inverse correlation has been noted between reaction yields and selectivity at $C \beta$, resulting in a precipitous drop in diastereoselectivity when reactions are run to high conversions. While there is a clear tradeoff between conversion and diastereoselectivity for LTTAcatalyzed reactions, we have demonstrated that a balance can be struck to deliver stereo-enriched products with a moderate sacrifice in isolated yields. Notably, the enantioselectivity of these transformations is quite high $(>99 \%$ ee). Phenylserine analogs generated under these conditions include $m$-bromophenylserine (19) and gram scale reactions to produce $p$-bromophenylserine (20) and p-chloro-phenylserine (18). To unambiguously assign the absolute configuration of $\mathrm{ObiH}-$ generated product 18, the compound was recrystallized and characterized by small-molecule $\mathrm{X}$-ray diffraction. This experiment confirmed that the major product of ObiH-catalyzed aldol addition was the $(2 S, 3 R)$, 'threo' isomer, which matched the observed stereochemistry of the native product 9 in obafluorin biosynthesis. ${ }^{[28,38]}$ 


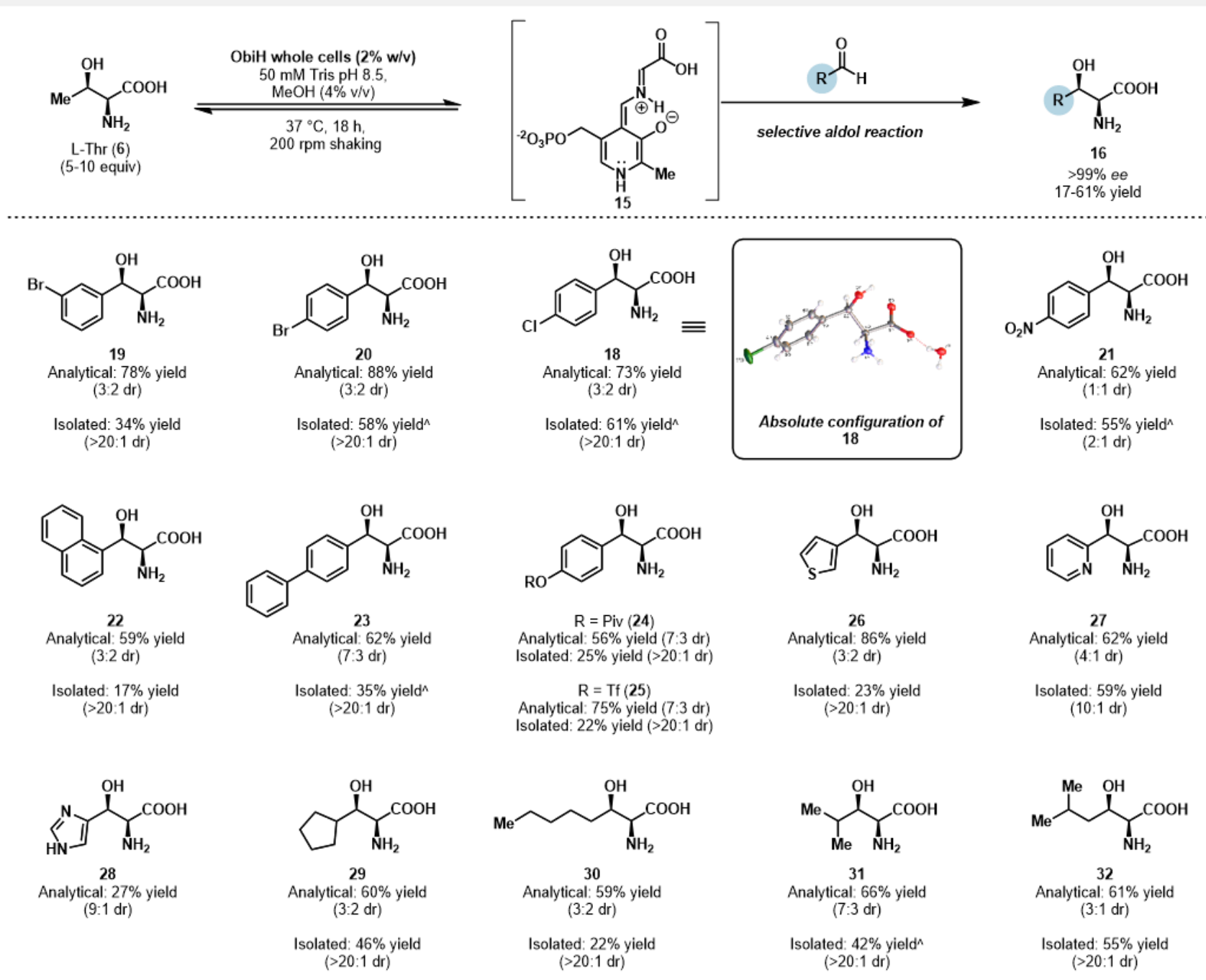

Figure 2. Analytical and preparative-scale synthesis of $\beta$-hydroxy- $\alpha$-amino acids by ObiH. Reactions were performed using $20 \mathrm{mM}$ aldehyde, $100 \mathrm{mM} \mathrm{L}$ $\mathrm{Thr}, 50 \mathrm{mM}$ Tris $\mathrm{pH} 8.5$ and $2 \% \mathrm{ObiH}$ wet whole cells, with $4 \%(\mathrm{v} / \mathrm{v}) \mathrm{MeOH}$ as co-solvent. Preparative scale reactions were incubated at $37{ }^{\circ} \mathrm{C}$ for $18 \mathrm{~h}$ before quench with 1 volume equivalent of MeCN, followed by freeze-thaw and centrifugation to remove cell debris. Purification was achieved using a Biotage purification system via reverse-phase chromatography. Yields are reported as isolated product mass after lyophilization. ${ }^{1} \mathrm{H}$ NMR hydration analysis was used to correct yield values for excess water. Analytical scale product yields determined by UPLC-PDA-MS following derivatization with Marfey's reagent. ^Reaction conducted on gram-scale.

To further probe the substrate scope and scalability of ObiH-catalyzed aldol reactions, additional benzaldehyde substrates were tested. Reactions with $p$-nitrobenzaldehyde on analytical and preparative scale indicated good yields, but low diastereoselectivity, delivering $p$-nitro-phenylserine (21) on gram scale. This molecule is an intermediate in the synthesis of the antibiotic chloramphenicol (4), as well as a precursor to other non-standard amino acids, such as $p$-amino-phenylserine.

We considered a reaction with 4-fluoro-3nitrobenzaldehyde, (see Figure S1) which would yield an intermediate in the recent total synthesis of vancomycin, a peptide antibiotic. ${ }^{[39]}$ However, the highly electrophilic aldehyde starting material condensed with Thr, precluding effective entry into the active site. A recent directed evolution campaign generated TAs that can catalyze a selective aldol reaction with electron deficient benzaldehydes, and we were therefore less compelled to study this reaction further. ${ }^{[25]}$ Instead, to further probe the limits of the ObiH active site, we sought to challenge the enzyme with sterically bulky aromatic aldehydes to generate p-napth-1-ylserine (22) and biphenylserine (23) (Figure 2).
Preparative scale yields for these products were reduced relative to reactions with less bulky benzaldehydes and may be limited by their poor solubility in aqueous conditions. Nevertheless, these reactions show that bulky $\beta$-hydroxy amino acids can be isolated with high diastereselectivity (>20:1), delivering these challenging products in a scalable manner.

In addition to the variety of phenylserine analogs that were produced using this method, we sought to generate $\beta$-hydroxy- $\alpha$ amino acids which could be used directly in the synthesis of natural products and pharmaceuticals. For example, $\beta$ hydroxytyrosine is an nsAA found in several cyclic peptide natural products, including the antibiotic hypeptin (Figure 1, 1). ${ }^{[7]}$ We initially attempted to directly generate $\beta$-hydroxytyrosine via an ObiH-catalyzed aldol reaction with 4-hydroxybenzaldehyde, but observed no product formation. This lack of reactivity has been mirrored in other TA systems, but no strategy to access these products has been put forth. ${ }^{[25]}$ We reasoned that, because the $\mathrm{pKa}$ of the phenol is low relative to the $\mathrm{pH}$ of the reaction, the predominant form in solution is the highly electron rich phenolate. Consequently, this molecule is an intrinsically 
inert substrate for biocatalytic aldol reactions. We anticipated that protection of the phenolic group on this substrate with an electron-withdrawing substituent would prevent ionization and increase the electrophilicity of the benzaldehyde, enabling a productive catalytic reaction. This hypothesis was confirmed in reactions with pivaloyl-protected 4-hydroxybenzaldehyde, generating the protected $\beta$-hydroxytyrosine 24 in $25 \%$ yield on milligram scale. As was previously observed, diastereoselectivity was improved in comparison to analytical scale conditions, owing to reduced yields in large scale reactions. This protection strategy was also successful with a trifluoromethylsulfonyl protected aldehyde, delivering the triflated $\beta$-hydroxytyrosine $\mathbf{2 5}$ in $22 \%$ yield. Through this simple substrate modification procedure, we generated a pair of aldehydes that could undergo productive catalysis with $\mathrm{ObiH}$, enabling efficient and selective access to protected $\beta$-hydroxytyrosine. Furthermore, the protection as a triflate provides a useful functional handle compatible for downstream diversification through crosscoupling reactions. ${ }^{[40]}$

Heterocyclic amino acids possess broad potential to be used as synthetic building blocks in the production of pharmaceuticals. ${ }^{[41]}$ Notably, access to heterocyclic $\beta$-hydroxy amino acids has also been a challenge for TA catalysis. ${ }^{[42]}$ In pursuit of a novel route to generate these valuable intermediates, we attempted ObiH aldol addition with heterocyclic aldehydes. These transformations proceeded smoothly, delivering pyridine2-yl-serine (27) in good yield (59\%) and with moderate diastereoselectivity $(10: 1 \mathrm{dr})$, as well as 3-thienylserine (26) in diminished yield (23\%). As was observed with benzaldehyde substrates, higher diastereoselectivities were obtained in the lower yielding preparative scale transformations, indicating that this phenomenon persists for reactions with heterocyclic aldehyde substrates. We further envisioned that $\mathrm{ObiH}$ could be used to generate $\beta$-hydroxy-tryptophan through reactions with indole-3-carboxaldehyde (Figure S1). However, no amino acid product was observed in these reactions, potentially due to the electron-rich nature of the aldehyde. Reactions with imidazole4-carboxyaldehyde gave rise to a prominent peak in the UPLCMS indicative of synthesis of $\beta$-hydroxy-histidine (28). However, the highly polar nature of this amino acid hindered its isolation. Nevertheless, Marfey's analysis indicates that $\mathrm{ObiH}$ can generate 28 in $27 \%$ yield based on UPLC analysis with 9:1 diastereoselectivity (Figure 2).

Following successful preparative-scale synthesis of diverse aromatic and heterocyclic amino acids using $\mathrm{ObiH}$, we were inspired to explore the abilities of the enzyme to catalyze reactions with aliphatic aldehydes. Preparative scale reactions with branched-chain aliphatic aldehydes proceeded smoothly, with excellent diastereomeric ratios (>20:1). As with all $\mathrm{ObiH}$ reactions performed in this study, we again observed reduced diastereoselectivity in higher yielding analytical scale reactions. Nevertheless, we isolated $\beta$-hydroxy-cyclopentanyl product 29 in $46 \%$ yield. Productive catalysis was also observed with isobutyraldehyde, delivering $\beta$-hydroxyleucine on gram scale (31, $42 \%$ yield) and we obtained $\beta$-hydroxyhomoleucine (32) in $55 \%$ yield. Impressively, the straight-chain hexanyl product $\mathbf{3 0}$ was also generated with high diastereoselectivity (>20:1), albeit with a reduced yield of $22 \%$, indicating that $\mathrm{ObiH}$ can undergo productive catalysis with sterically bulky, unstrained aldehydes. However, conversion was not observed in reactions with larger straight-chain aldehyde dodecanal, suggesting that there are practical limitations in substrate size for $\mathrm{ObiH}$ catalyzed reactions. The success of $\mathrm{ObiH}$ in generating a variety of aliphatic $\beta$-hydroxy amino acids is a significant advance, as biocatalytic access to these motifs has generally been limited to a few examples of threonine aldolases with low to moderate diastereoselectivities. ${ }^{[43,44]}$ While biosynthetic strategies which involve late stage selective hydroxylation have found success in generating aliphatic $\beta$-hydroxy alpha amino acids, this approach is highly substrate specific and precludes a generalized route to simultaneously install a diverse variety of side chains and the $\beta$ hydroxyl group. ${ }^{[18,19]} \mathrm{ObiH}$-catalyzed aldol reactions therefore offer a streamlined enzymatic route to access diverse $\beta$-hydroxy amino acids.

Inspired by the success of ObiH in producing a variety of $\beta$-hydroxy amino acids, we sought to challenge the enzyme with a large, fluorescent aldehyde derived from the valuable probe molecule BODIPY. ${ }^{[45]}$ An initial whole cell $\mathrm{ObiH}$ reaction was performed on milligram scale to generate BODIPY-containing $\beta$ hydroxy amino acid 34 (Figure 3). Aldehyde 33 underwent productive catalysis with $\mathrm{ObiH}$, generating product $\mathbf{3 4}$ in poor yield ( 1\%). We improved the yields by using purified $\mathrm{ObiH}$ as catalyst and by the addition of $10 \%$ DMSO as co-solvent to increase the solubility of $\mathbf{3 3}$. These changes improved the yield to $7 \%$ with pristine diastereoselectivity $(>20: 1)$. While protein engineering would be needed to further increase the reactivity of ObiH with this aldehyde, such efforts are straightforward once initial activity is demonstrated, which we provide here. Fluorescence spectra showed that product $\mathbf{3 4}$ exhibits characteristic fluorescence after excitation at $501 \mathrm{~nm}$ and

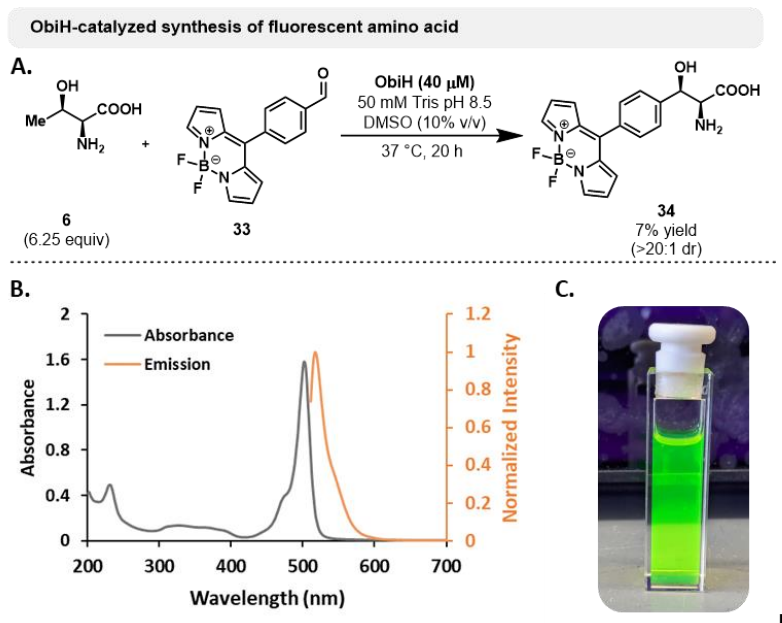

Figure

3. A. ObiH-catalyzed synthesis of BODIPY-containing $\beta$-hydroxy- $\alpha$-amino acid 34. Reactions were performed using $20 \mathrm{mM}$ aldehyde, $125 \mathrm{mM} \mathrm{L-Thr}$, $50 \mathrm{mM}$ Tris $\mathrm{pH} 8.5$ and $40 \mu \mathrm{M}$ purified ObiH with $10 \%$ (v/v) DMSO as cosolvent. Reactions were incubated at $37^{\circ} \mathrm{C}$ for $20 \mathrm{~h}$ before quenching with 1 volume equivalent of $\mathrm{MeCN}$ and centrifugation to remove protein debris. Purification was achieved using a Biotage purification system via reversephase chromatography. Yields are reported as isolated product mass after lyophilization. ${ }^{1} \mathrm{H}$ NMR hydration analysis was used to correct yield values for excess water. B. Absorbance and emission spectra of $20 \mu \mathrm{M}$ amino acid 34 dissolved in $\mathrm{MeOH}$. C. $20 \mu \mathrm{M}$ of amino acid $\mathbf{3 4}$ in $\mathrm{MeOH}$ under UV light. 
<smiles>CC(=O)C(N)[C@@H](N)C(=O)O</smiles>

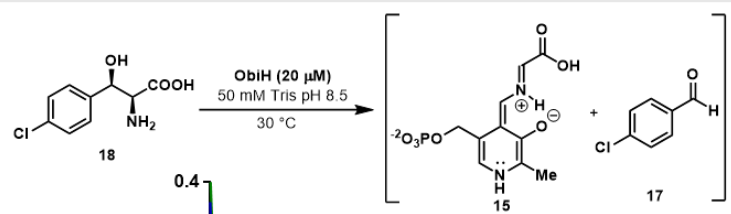
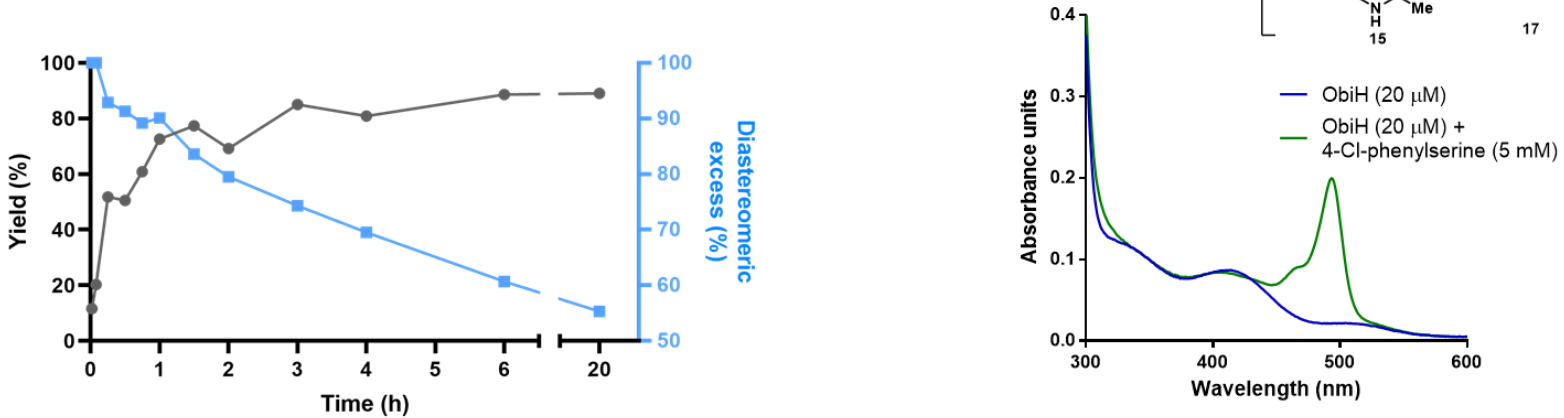

C. ObiH-catalyzed racemization of 4-chloro-phenylserine (18)

D. ObiH active site model with 4-chloro-phenylserine (18) bound
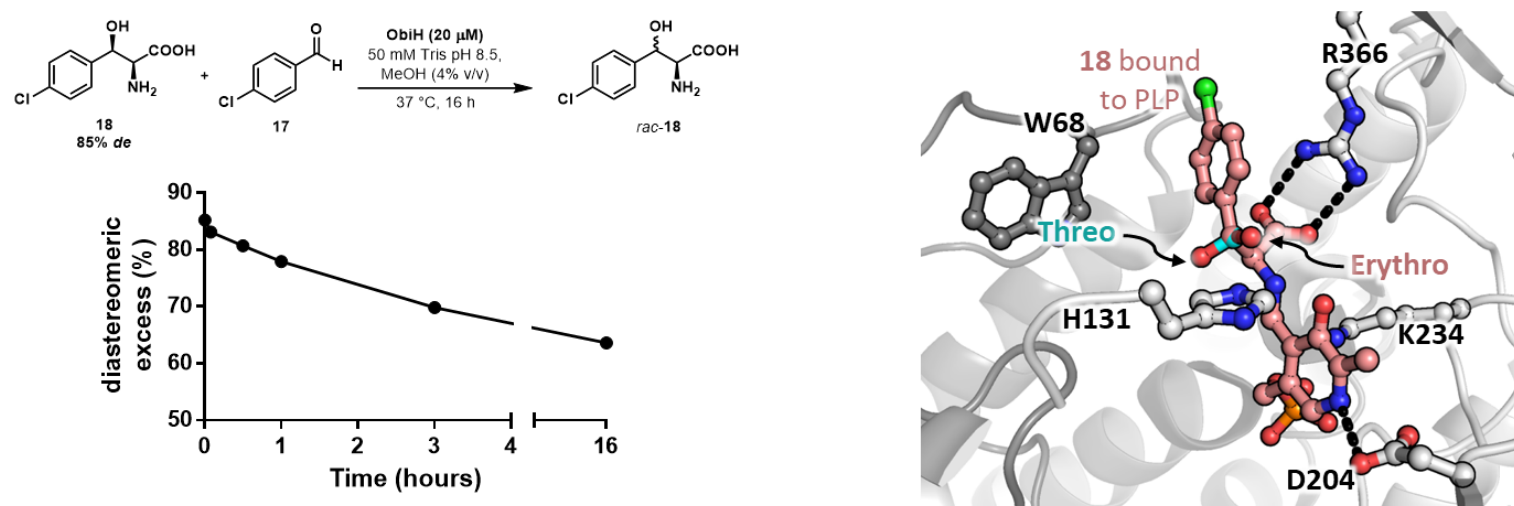

Figure 4. A. Plot of diastereoselectivity of ObiH-catalyzed reaction with 4-chlorobenzaldehyde versus conversion at varying timepoints. B. UV-visible spectrum following titration of $\mathrm{ObiH}$ with 4-chloro-phenylserine (18) demonstrating glycyl quinonoid $(15)$ formation $\left(\lambda_{\text {max }}=494 \mathrm{~nm}\right)$. C. ObiH-catalyzed racemization of diastereomerically-enriched 4-chloro-phenylserine (18). D. ObiH active site model with 4-chloro-phenylserine bound to PLP as the external aldimine (EAex). The active site is at the dimer interface and individual monomers are colored in light and dark grey. Active site residues are shown as sticks. The erythro isomer is colored salmon and the threo isomer is colored cyan. Hydrogen bonds are shown as black dashes.

emission at $517 \mathrm{~nm}$ (Figure 3). We anticipate that this amino acid could be a valuable tool in chemical biology applications. This reaction further demonstrates the potential of $\mathrm{ObiH}$ to react with a range of aldehydes to generate functional amino acid products. Despite some limitations in its native reactivity, we used $\mathrm{ObiH}$ to generate numerous benzylic, heterocyclic and aliphatic $\beta$-hydroxy amino acids on preparative scale. These efforts demonstrate the native promiscuity of $\mathrm{ObiH}$ toward a variety of inexpensive aldehydes, selectively producing important synthetic precursors in one step. Additionally, this biocatalytic approach enables the use of Thr as an inexpensive substrate without any pre-functionalization, which is a considerable advantage over previous synthetic approaches. However, observation of an inverse correlation between yield and diastereoselectivities in these transformations spurred further mechanistic questions about this common phenomenon in LTTAs.

To characterize catalyst behavior throughout the reaction, we measured a time course of the $\mathrm{ObiH}$ reaction with 4chlorobenzaldehyde. This reaction yielded two well-resolved diastereomers, enabling direct comparison (via UPLC) of the observed yield of product $\mathbf{1 8}$ and diastereomeric ratios for each timepoint. At early timepoints, the diastereomeric excess was high, indicating that $\mathrm{ObiH}$ exhibits superb diastereoselectivity.
Analysis of later time points showed a notable reduction in the diasteromeric excess of product $\mathbf{1 8}$ as yield increased beyond $50 \%$ (Figure $4 \mathrm{~A}$ ). Based on our previous studies of the mechanism of ObiH transaldolase activity, ${ }^{[32]}$ we hypothesized that this drop in the diastereomeric excess is caused by the reversibility of the transaldolase reaction and product reentry into the catalytic cycle.

To demonstrate that product can reenter the catalytic cycle and generate glycyl-quinonoid intermediate 15 , we performed a UV-vis experiment (Figure 4B) in which $\mathrm{ObiH}$ was mixed with $p$ chloro-phenylserine (18). In this experiment, following the addition of $5 \mathrm{mM} \mathrm{18}$, we observed a shift from the internal aldimine:PLP signal $(405 \mathrm{~nm})$ to a new peak at $494 \mathrm{~nm}$, corresponding to stabilized glycyl-quinonoid intermediate ${ }^{15} .^{[32]}$ This same intermediate was also observed upon titration of ObiH with native transaldolase substrate L-Thr, suggesting that the 4-chloro-phenylserine product can indeed reenter the catalytic cycle through retro-aldol cleavage. ${ }^{[32]}$ For direct evidence of product reentry leading to diastereomeric erosion, we monitored an $\mathrm{ObiH}$ reaction with $p$-chloro-phenylserine under turnover conditions in the presence of 4-chlorobenzaldehyde (17) and observed a slow decrease in diastereomeric excess over time, beginning with a de value of $82 \%$ and ending with a de of $64 \%$ after $18 \mathrm{~h}$ (Figure $3 \mathrm{C}$ ). These experiments confirm 
that ObiH product 18 can indeed reenter the catalytic cycle to produce an on-cycle quinonoid intermediate and that this behavior leads directly to reduced diastereomeric excess for ObiH products. These data are consistent with observations that high yielding reactions with $\mathrm{ObiH}$ exhibit reduced diastereoselectivity for all tested substrates.

Based on these observations, we rationalized that product reentry leads to scrambling at the $\beta$-position through iterative cycles of retro-aldol product cleavage, followed by the forward aldol reaction with 4-chlorobenzaldehyde. Because the major isomer is at a higher concentration at later time points, it will preferentially re-enter the active site and be broken down, only to be re-formed as a mixture of threo and erythro isomers. In this way, the kinetically disfavored erythro isomer slowly accumulates during as the diastereomeric ratio shifts towards an equilibrium ratio. Notably, the stereochemistry at $\mathrm{Ca}$ is maintained within the limits of detection during all of the experiments described here.

To probe the structural basis for the diastereoselectivity of ObiH, we turned to the recently described structure at $1.66 \AA$ (PDB ID: 7K34). ${ }^{[32]}$ This structure is of the internal aldimine state of the enzyme and, despite extensive efforts, we were unable to determine a substrate-bound structure under these conditions. We previously turned to molecular dynamics, which provided a plausible structure of $\mathrm{ObiH}$ with $\mathrm{Thr}$ bound as the external aldimine. ${ }^{[32]}$ Here, we considered how a $\beta$-arene would fit into the active site and found that it was a highly constricted environment. Only a single staggered rotamer could be formed without a significant steric clash with protein backbone (Figure S1). While it is possible that some extensive conformational rearrangement occurs upon substrate binding, these areas of contact were stable under the conditions of our previous molecular dynamics simulations. We therefore hypothesize that the bulky substrates described here bind in a single, common orientation extending towards Trp68, which is found on a highly flexible loop (Figure 4D). In this model the preferred, on-pathway, mode of binding delivers the si-face of the electrophile to the $\mathrm{E}\left(\mathrm{Q}^{\mathrm{Gly}}\right)$ nucleophile and aldol addition gives rise to the threo product.

A. Chemical and enzymatic modification of $\mathrm{ObiH}$-generated $\beta$-hydroxyleucine (36)

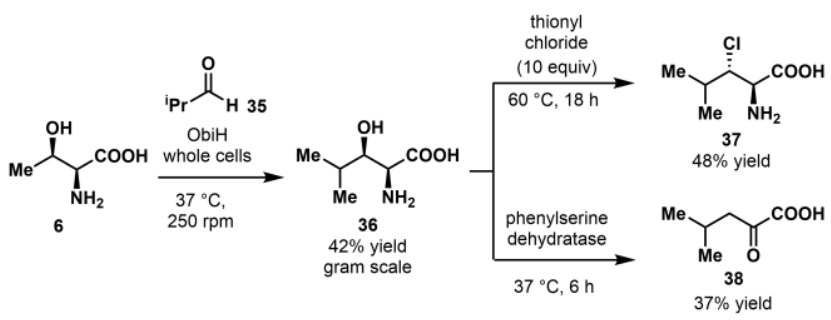

B. Representative natural products possessing the $\beta$-chloroleucine motif

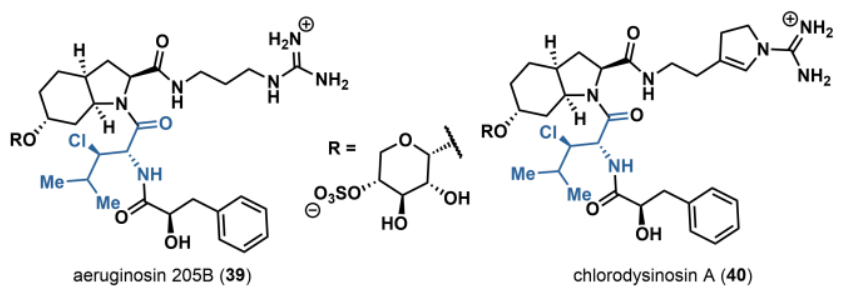

Figure 5. Downstream chemical modifications of ObiH-generated $\beta$ hydroxyleucine.
We also sought to further highlight the synthetic utility of ObiH-generated products through downstream functionalization reactions to access important amino acid motifs. For example, $\beta$ chloroleucine is a nsAA found in the aerugenosins (Figure 5B, see 39 and 40), a class of peptide natural products which act as protease inhibitors. ${ }^{[46]}$ Following gram scale ObiH-catalyzed generation of threo- $\beta$-hydroxyleucine (36), we subjected the purified material to conditions for $\beta$-chlorination. Amino acid $\mathbf{3 6}$ was dissolved in neat thionyl chloride (10 equiv) and heated to $60{ }^{\circ} \mathrm{C}$ overnight. Following a quench to hydrolyze the resulting acid chloride material, erythro- $\beta$-chloroleucine (37) was isolated in a $42 \%$ yield and high diastereoselectivity. This synthesis leverages the selectivity of $\mathrm{ObiH}$ in asymmetric $\mathrm{C}-\mathrm{C}$ bond formation, enabling diastereoselective access to halogenated amino acids.

Finally, we aimed to demonstrate the utility of ObiH derived $\beta$-hydroxy amino acids as precursors to aliphatic $\alpha$-ketoacids (Figure 5A). The phenylserine dehydratase from Ralstonia pickettii was previously shown to react with a range of phenyl serine analogs. Here we show this scope can be extended beyond aromatic amino acids by developing a one-pot, telescoped sequence for generating $a$-keto acid $\mathbf{3 8}$ directly from L-Thr (6) and isobuyraldehyde (36). ${ }^{[47]}$ a-keto acid 38 was formed in $37 \%$ yield through this sequence. Such $\alpha$-keto acid products are highly desirable and have found use as intermediates in biocatalytic cascade reactions, acylating agents in organic synthesis and as precursors for the synthesis of biofuels and pharmaceuticals. ${ }^{[48-50]}$ Through these functionalization reactions, we have demonstrated the diverse products which can be accessed in an efficient manner through ObiH-catalyzed transformations.

\section{Conclusions}

Here we have demonstrated the synthetic utility of ObiH for the diastereoselective production of diverse and valuable $\beta$-hydroxy$\alpha$-amino acids from inexpensive starting materials. These molecules represent an important starting point for the synthesis of natural products and pharmaceuticals, and can be probes for biological systems. Recognizing the value of a transformation that directly produces $\beta$-hydroxy- $\alpha$-amino acids, we sought to efficiently generate a variety of these materials through preparative-scale reactions using whole $E$. coli cells, including several transformations on gram scale. We have characterized the native reactivity of $\mathrm{ObiH}$ toward a panel of aldehydes, probing the abilities and limitations of $\mathrm{ObiH}$-catalyzed aldolase reactions. This substrate scope analysis was supported by detailed characterization of $\mathrm{ObiH}$ diastereoselectivity and underpinning mechanistic implications of observed selectivity trends under analytical and preparative scale conditions. Through these efforts, we have developed an efficient route to accessing important organic building blocks, including downstream functionalization reactions to directly synthesize $\beta$ halogenated amino acids and $\alpha$-keto acids. Based on the simplicity and versatility of this reaction platform, we advance $\mathrm{ObiH}$ as a useful biocatalyst for selective $\mathrm{C}-\mathrm{C}$ bond formation and we anticipate that this enzyme will serve as a new and effective implement in the organic chemist's toolbox. 


\section{Acknowledgements}

This work was supported by the Office of the Vice Chancellor for Research and Graduate Education at the University of Wisconsin-Madison with funding from the Wisconsin Alumni Research Foundation and the NIH DP2-GM137417 to A.R.B.; Morgridge Institute for Research - Metabolism Theme Fellowship to P.K.; NIH The NMR spectrometers were supported by the Bender Fund. The purchase of the Bruker D8 VENTURE Photon III X-ray diffractometer was partially funded by NSF Award \#CHE-1919350 to the UW-Madison Department of Chemistry.

We acknowledge the invaluable support, assistance and advice from our colleagues in the Buller group.

Keywords: Biocatalysis, $\mathrm{C}-\mathrm{C}$ bond formation, threonine transaldolase

[1] M. A. T. Blaskovich, J. Med. Chem. 2016, 59, 10807-10836. [2] C.-I. Lin, R. M. McCarty, H. Liu, Angew. Chemie Int. Ed. 2017, 56, 3446-3489.

[3] M. Hönig, P. Sondermann, N. J. Turner, E. M. Carreira, Angew. Chemie - Int. Ed. 2017, 56, 8942-8973.

[4] L. E. Zetzsche, A. R. H. Narayan, Nat. Rev. Chem. 2020, 4, 334-346.

[5] E. Watkins-Dulaney, S. Straathof, F. Arnold, ChemBioChem 2021, 22 5-16.

[6] M. A. Huffman, A. Fryszkowska, O. Alvizo, M. Borra-Garske, K. R Campos, K. A. Canada, P. N. Devine, D. Duan, J. H. Forstater, S. T. Grosser, H. M. Halsey, G. J. Hughes, J. Jo, L. A. Joyce, J. N. Kolev, J. Liang, K. M. Maloney, B. F. Mann, N. M. Marshall, M. McLaughlin, J. C. Moore, G. S.

Murphy, C. C. Nawrat, J. Nazor, S. Novick, N. R. Patel, A. Rodriguez-Granillo, S. A. Robaire, E. C. Sherer, M. D. Truppo, A. M. Whittaker, D. Verma, L. Xiao, Y. Xu, H. Yang, Science (80-. ). 2019, 366, 1255-1259.

[7] M. Crüsemann, D. A. Wirtz, K. C. Ludwig, M. Arts, C. E. Marx, S

Krannich, P. Barac, S. Kehraus, M. Josten, B. Henrichfreise, A. Müller, G. M.

König, A. J. Peoples, A. Nitti, A. L. Spoering, L. L. Ling, K. Lewis, T. Schneider, Angew. Chemie Int. Ed. 2021, 1-9.

[8] H. Maki, K. Miura, Y. Yamano, Antimicrob. Agents Chemother. 2001, $45,1823-1827$.

[9] M. D. Corbett, B. R. Chipko, Antimicrob. Agents Chemother. 1978, 13, 193-198.

[10] T. Ooi, M. Taniguchi, M. Kameda, K. Maruoka, Angew. Chemie - Int. Ed 2002, 41, 4542-4544.

[11] Y. Singjunla, J. Baudoux, J. Rouden, Org. Lett. 2013, 15, 5770-5773.

[12] R. Thayumanavan, F. Tanaka, C. F. Barbas, Org. Lett. 2004, 6, 35413544 .

[13] S. Mettath, G. S. C. Srikanth, B. S. Dangerfield, S. L. Castle, D. L. M. R Rev, 2004, 6489-6492.

[14] S. Vera, A. Vázquez, R. Rodriguez, S. Del Pozo, I. Urruzuno, A. de

Cózar, A. Mielgo, C. Palomo, J. Org. Chem. 2021, 86, 7757-7772.

[15] D. A. Evans, J. M. Janey, N. Magomedov, J. S. Tedrow, Assessment 2001, 1936-1940.

[16] M. J. Cryle, A. Meinhart, I. Schlichting, J. Biol. Chem. 2010, 285, 24562-24574.

[17] H. Chen, M. G. Thomas, S. E. O'Connor, B. K. Hubbard, M. D. Burkart, C. T. Walsh, Biochemistry 2001, 40, 11651-11659.

[18] H. Renata, E. Shimizu, C. R. Zwick, Tetrahedron 2021, 90, 132190.

[19] C. R. Zwick, M. B. Sosa, H. Renata, 2021, 0-6.

[20] T. Kimura, V. P. Vassilev, G.-J. Shen, C.-H. Wong, J. Am. Chem. Soc 1997, 119, 11734-11742.

[21] M. L. Di Salvo, S. G. Remesh, M. Vivoli, M. S. Ghatge, A. Paiardini, S D'Aguanno, M. K. Safo, R. Contestabile, FEBS J. 2014, 281, 129-145.

[22] K. Fesko, Appl. Microbiol. Biotechnol. 2016, 100, 2579-2590.

[23] Q. Chen, X. Chen, J. Feng, Q. Wu, D. Zhu, Y. Ma, ACS Catal. 2019, 9, 4462-4469.

[24] D. Recombinant, L. Aldolases, T. Kimura, V. P. Vassilev, G.-J. Shen, C.-H. Wong, T. Scripps, N. Torrey, P. Road, L. Jolla, J. Am. Chem. Soc. 1997, 7863119, 11734-11742.

[25] W. Zheng, H. Yu, S. Fang, K. Chen, Z. Wang, X. Cheng, G. Xu, L. Yang, J. Wu, ACS Catal. 2021, 11, 3198-3205.

[26] S. L. Goldberg, A. Goswami, Z. Guo, Y. Chan, E. T. Lo, A. Lee, V. Chi Truc, K. J. Natalie, C. Hang, L. T. Rossano, M. A. Schmidt, Org. Process Res. \&amp; Dev. 2015, 19, 1308-1316.

[27] J. E. Schaffer, M. R. Reck, N. K. Prasad, T. A. Wencewicz, Nat. Chem. Biol. 2017, 13, 737-744.
[28] T. A. Scott, D. Heine, Z. Qin, B. Wilkinson, Nat. Commun. 2017, 8, 15935

[29] D. F. Kreitler, E. M. Gemmell, J. E. Schaffer, T. A. Wencewicz, A. M.

Gulick, Nat. Commun. 2019, 10,1-13.

[30] L. Xu, L. C. Wang, B. M. Su, X. Q. Xu, J. Lin, Bioresour. Technol. 2020 310,123439

[31] N. Dückers, K. Baer, S. Simon, H. Gröger, W. Hummel, Appl. Microbiol. Biotechnol. 2010, 88, 409-424.

[32] P. Kumar, A. Meza, J. M. Ellis, G. A. Carlson, C. A. Bingman, A. R Buller, ACS Chem. Biol 2021, 16,95.

[33] S. Wang, H. Deng, Appl. Microbiol. Biotechnol. 2021, 2, 3507-3520.

[34] J. Wachtmeister, D. Rother, Curr. Opin. Biotechnol. 2016, 42, 169-177.

[35] S. A. Baker Dockrey, T. J. Doyon, J. C. Perkins, A. R. H. Narayan,

Chem. Biol. Drug Des. 2019, 93, 1207-1213.

[36] F. Garzón-Posse, L. Becerra-Figueroa, J. Hernández-Arias, D. GambaSánchez, Molecules 2018, 23, DOI 10.3390/molecules23061265

[37] L. Xu, L. C. Wang, X. Q. Xu, J. Lin, Catal. Sci. Technol. 2019, 9, 59435952

[38] J. E. Schaffer, M. R. Reck, N. K. Prasad, T. A. Wencewicz, Nat. Chem. Biol. 2017, 13, 737-744.

[39] M. J. Moore, S. Qu, C. Tan, Y. Cai, Y. Mogi, D. J. Keith, D. L. Boger, J. Am. Chem. Soc. 2020, 142, 16039-16050.

[40] T. Willemse, W. Schepens, H. W. T. Van Vlijmen, B. U. W. Maes, S. Ballet, Catalysts 2017, 7, DOI 10.3390/catal7030074.

[41] M. Wang, K. P. Rakesh, J. Leng, W. Y. Fang, L. Ravindar, D. Channe

Gowda, H. L. Qin, Bioorg. Chem. 2018, 76, 113-129.

[42] S. F. Beaudoin, M. P. Hanna, I. Ghiviriga, J. D. Stewart, Enzyme

Microb. Technol. 2018, 119, 1-9.

[43] J. Steinreiber, K. Fesko, C. Mayer, C. Reisinger, M. Schürmann, H

Griengl, Tetrahedron 2007, 63, 8088-8093.

[44] J. Blesl, M. Trobe, F. Anderl, R. Breinbauer, G. A. Strohmeier, K. Fesko, ChemCatChem 2018, 10, 3453-3458.

[45] S. Hanessian, X. Wang, K. Ersmark, J. R. Del Valle, 2009, 3, 1-4.

[46] O. Heiwa, N. Shinji, M. Haruo, Biotechnol. Biochem. 2002, 66, 27552758

[47] Y. P. Xue, C. H. Cao, Y. G. Zheng, Chem. Soc. Rev. 2018, 47, 1516 1561.

[48] F. Penteado, E. F. Lopes, D. Alves, G. Perin, R. G. Jacob, E. J.

Lenardão, Chem. Rev. 2019, 119, 7113-7278.

[49] S. Atsumi, T. Hanai, J. C. Liao, Nature 2008, 451, 86-89. 


\section{Entry for the Table of Contents}

Insert graphic for Table of Contents here.

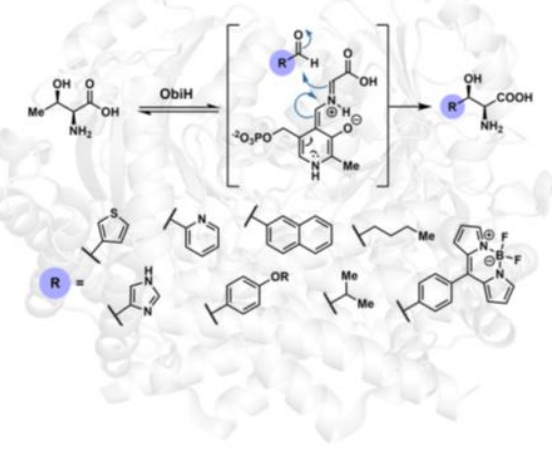

Insert text for Table of Contents here (max 450 characters with spaces). ObiH is an L-threonine transaldolase which catalyzes the diastereoselective synthesis of $\beta$-hydroxy- $\alpha$-amino acids. We have demonstrated the synthetic utility of this enzyme through preparative scale reactions to selectively generate a structurally diverse array of $\beta$-hydroxy- $\alpha$-amino acids, as well as downstream functionalization to generate important organic building blocks, such as $\beta$-chloro- $\alpha$-amino acids and $\alpha$-keto acids.

Institute and/or researcher Twitter usernames: @bullerlab 